\title{
NMR-based Quantum Mechanical Analysis Builds Trust and Orthogonality in Structural Analysis: the Case of a Bisdesmosidic Triglycoside as Withania somnifera Aerial Parts Marker
}

Sitaram Bhavaraju, ${ }^{1}$ David Taylor, ${ }^{1}$ Matthias Niemitz, ${ }^{2}$ David C. Lankin, ${ }^{3}$ Anton Bzhelyansky, ${ }^{1}$ Gabriel I. Giancaspro, ${ }^{1}$ Yang Liu, ${ }^{1}$ Guido F. Pauli ${ }^{3}$

${ }^{1}$ United States Pharmacopeial Convention, Rockville, Maryland 20852, United States

${ }^{2}$ NMR Solutions Oy, Kuopio, Northern Savonia 70110, Finland

${ }^{3}$ Department of Medicinal Chemistry and Pharmacognosy, University of Illinois at Chicago, Chicago, Illinois 60612, United States

\section{SUPPORTING INFORMATION}




\section{- TABLE OF CONTENTS}

No. Content Page

S1 NMR Spectra of Kaempferol-3-O-robinoside-7-O-glucoside (1): $\quad$ S-3

$1 \mathrm{D}{ }^{1} \mathrm{H}$ at 600 and $900 \mathrm{MHz}$

S2 NMR Spectrum of Kaempferol-3- $O$-robinoside-7- $O$-glucoside (1): $\quad$ S-6 $1 D^{13} \mathrm{C}$ NMR

S3 NMR Spectrum of Kaempferol-3-O-robinoside-7-O-glucoside (1): $\quad$ S-7 $2 \mathrm{D}{ }^{1} \mathrm{H},{ }^{1} \mathrm{H}-\mathrm{COSY}$

S4 NMR Spectrum of Kaempferol-3-O-robinoside-7-O-glucoside (1): $\quad$ S-8 $2 \mathrm{D}{ }^{1} \mathrm{H},{ }^{13} \mathrm{C}-\mathrm{HSQC}$

S5 NMR Spectrum of Kaempferol-3-O-robinoside-7-O-glucoside (1): $\quad$ S-9 $2 \mathrm{D}{ }^{1} \mathrm{H},{ }^{13} \mathrm{C}-\mathrm{HMBC}$

S6 NMR 2D ${ }^{1} \mathrm{H},{ }^{13} \mathrm{C}-\mathrm{HMBC}$ Spectral Comparison of Kaempferol-3-O- $\quad$ S-10 robinoside-7-O-glucoside (1) and Quercetin-3-O-robinoside-7-Oglucoside $\left({ }^{1} \mathrm{H} 400 \mathrm{MHz} /{ }^{13} \mathrm{C} 100 \mathrm{MHz}\right.$, DMSO- $d_{6}$ and $\left.\mathrm{D}_{2} \mathrm{O}\right)$

S7 Numerical Comparison of the HiFSA Profiles of 1 Obtained with PERCH S-11 and CT Software Packages 
S1. 1D ${ }^{1} \mathrm{H}$ NMR Spectra of Kaempferol-3-O-robinoside-7-O-glucoside (1): Experimental ${ }^{1} \mathrm{H} 600 \mathrm{MHz}$ Spectrum in DMSO-d 6 with $50 \%$ of $\mathrm{D}_{2} \mathrm{O}$ (top) and $900 \mathrm{MHz}$ HiFSA profile (bottom).

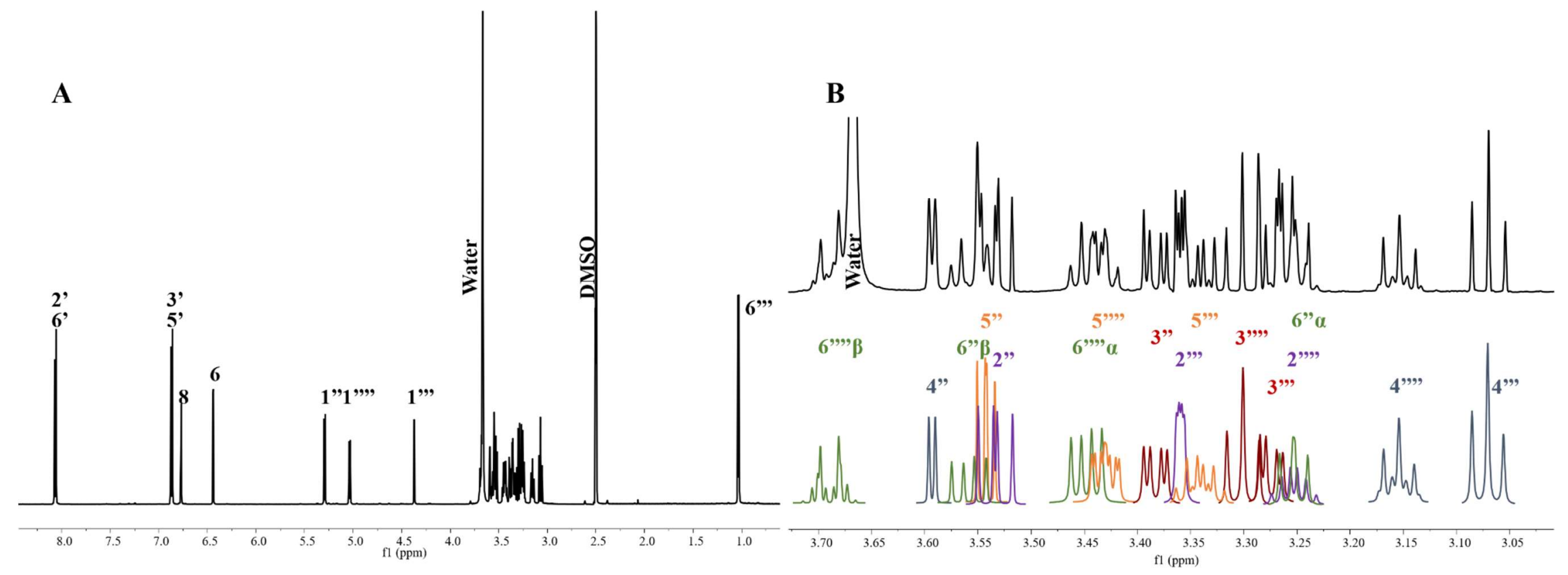

(A) Full experimental ${ }^{1} \mathrm{H}$ spectrum with all resonances of $\mathbf{1}$ and (B) zoomed-in spectral window from $3.0 \mathrm{ppm}$ to $3.9 \mathrm{ppm}$. To visualize severely overlapped signals, the calculated patterns (in color) and frequency of each resonances from 3.0 to $3.9 \mathrm{ppm}$ are shown in (B).

Shown below, the $900 \mathrm{MHz}$ 1D ${ }^{1} \mathrm{H}$ spectrum (C) and the HiFSA fingerprint derived from the $600 \mathrm{MHz}$ data (D) are identical and prove that even the further enhanced dispersion of the ultra-high magnetic field instrument cannot fully resolve signal overlap. While offering some improvement, the $900 \mathrm{MHz}$ spectrum does not provide more spectroscopic information than the $600 \mathrm{MHz}$ data. 
C

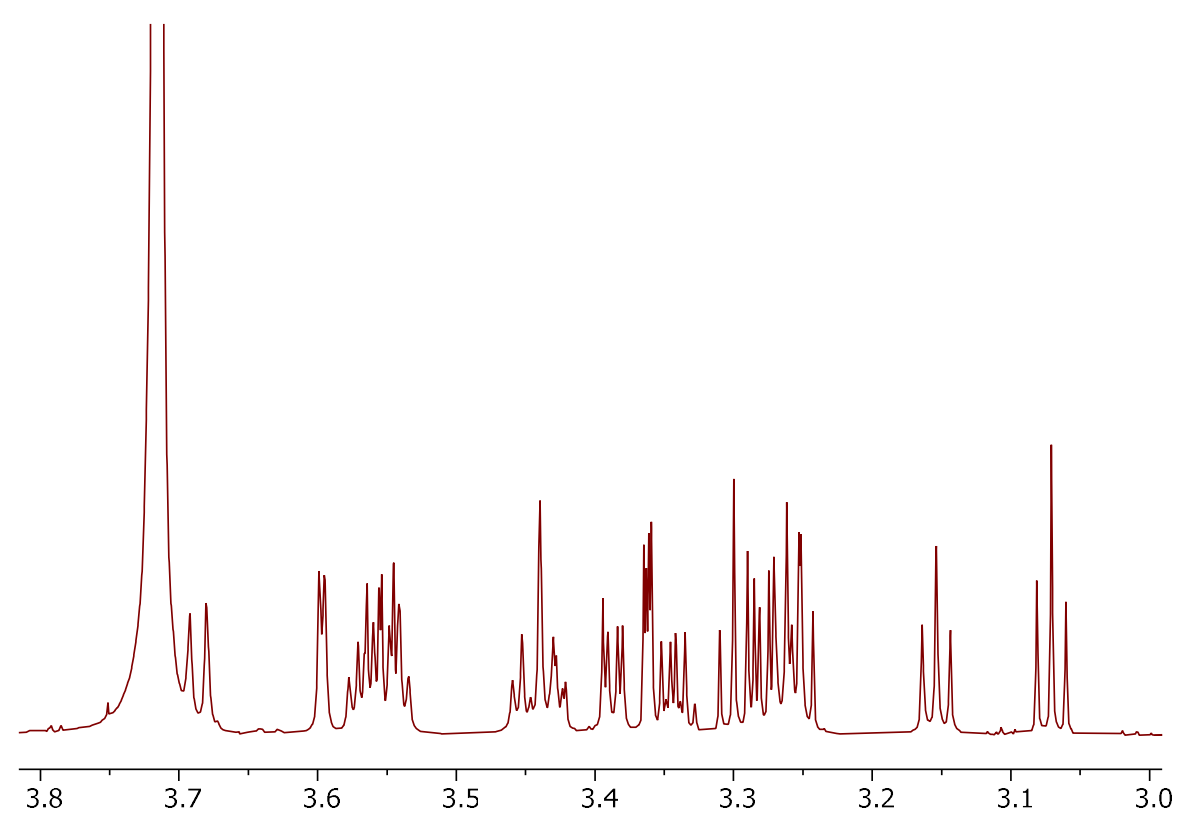




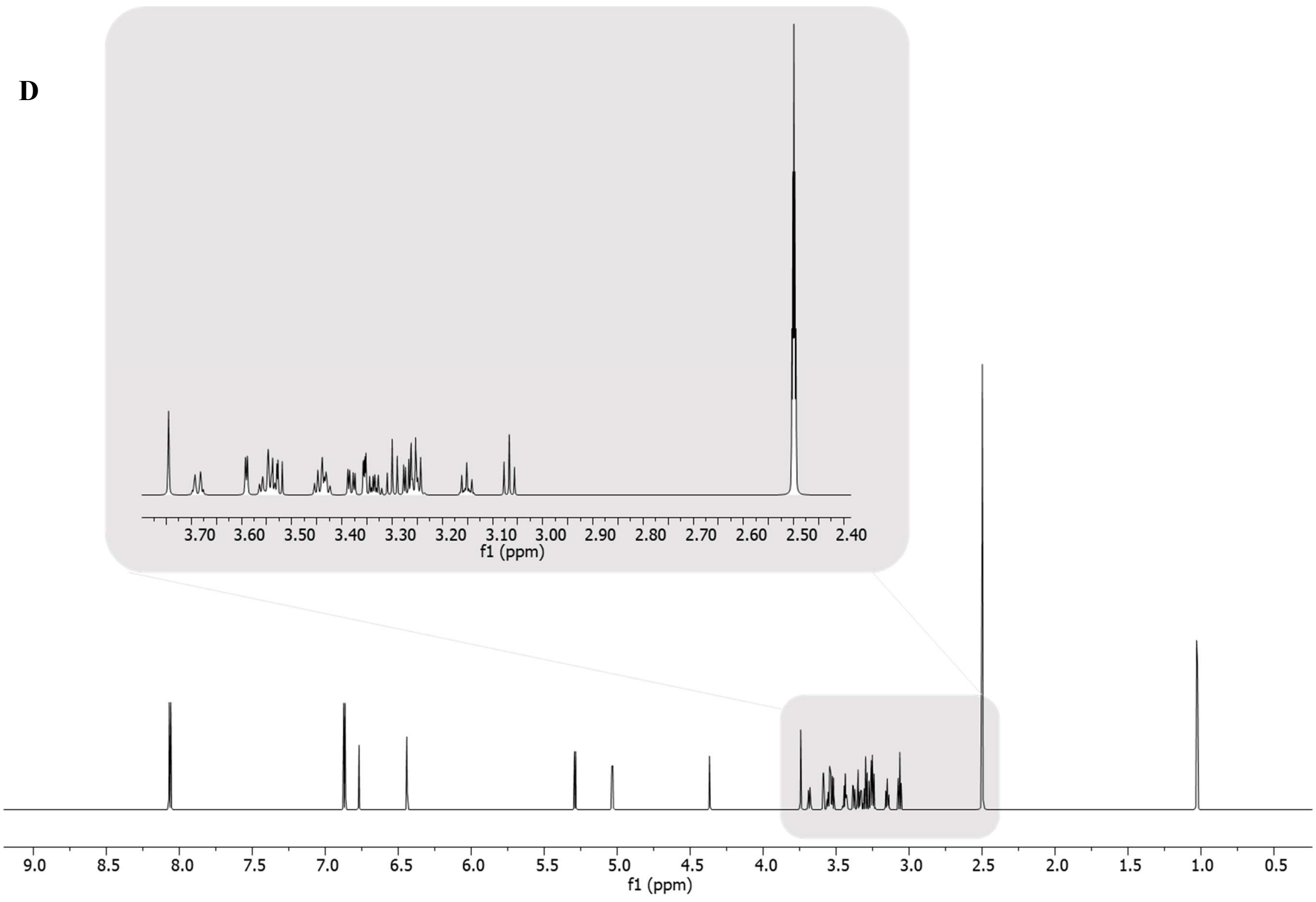


S2. 1D ${ }^{13} \mathrm{C}$ NMR Spectrum of Kaempferol-3-O-robinoside-7-O-glucoside (1) $\left({ }^{1} \mathrm{H} 600 \mathrm{MHz} /{ }^{13} \mathrm{C} 150 \mathrm{MHz}\right.$, DMSO-d 6 with $50 \%$ of $\left.\mathrm{D}_{2} \mathrm{O}\right)$.

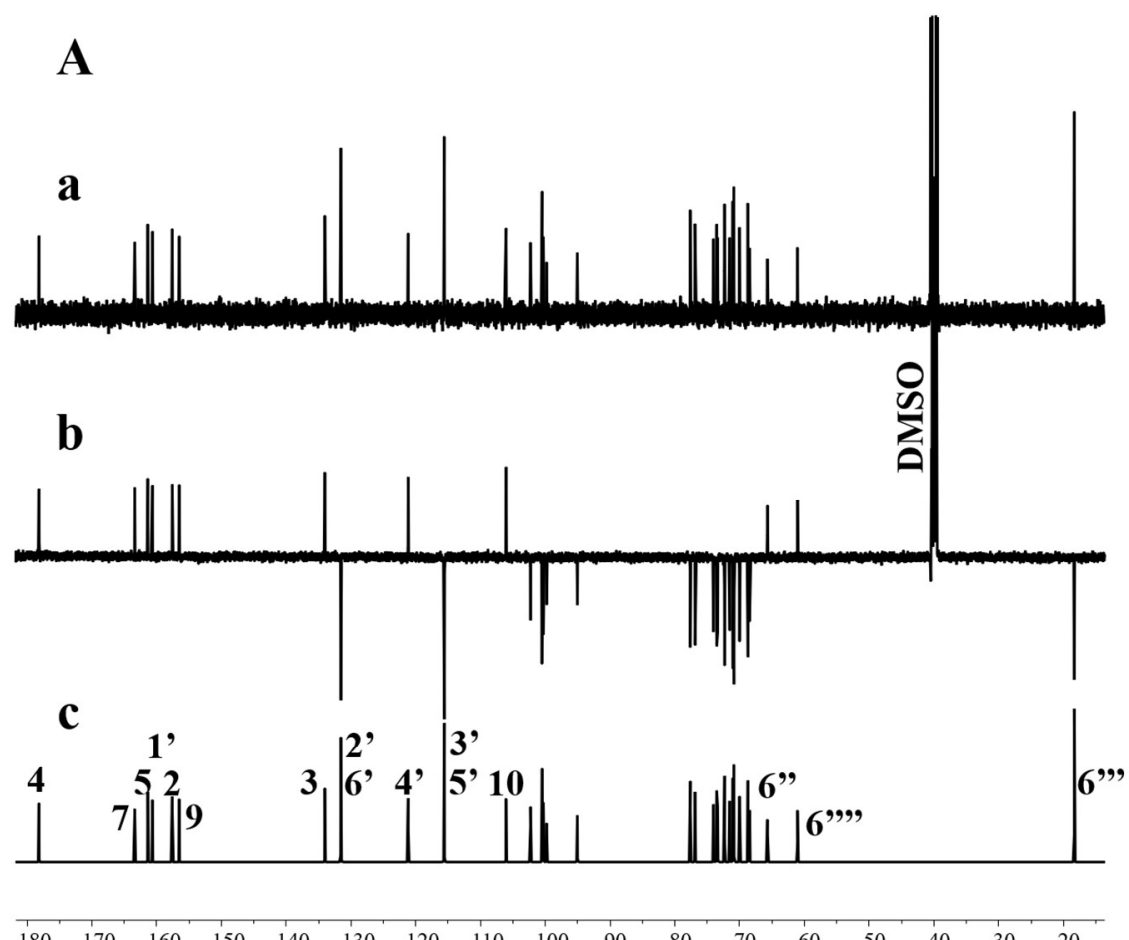

B

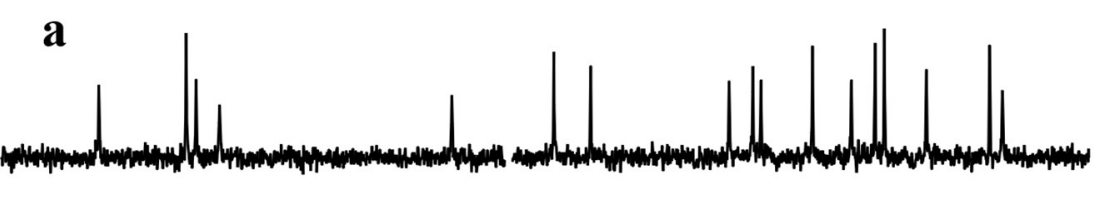

b

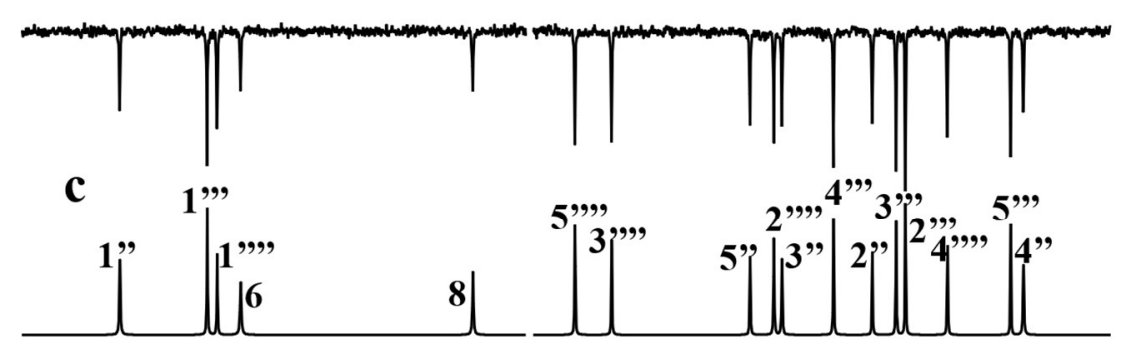

$\begin{array}{lllllllllllllllll}180 & 170 & 160 & 150 & 140 & 130 & 120 & 110 & 100 & 90 & 80 & 70 & 60 & 50 & 40 & 30 & 20 \\ \mathrm{fl}(\mathrm{ppm}) & & & & & & \end{array}$

$\begin{array}{lllllllllllllllllllllll}104 & 103 & 102 & 101 & 100 & 99 & 98 & 97 & 96 & 95 & 94 & 78 & 77 & 76 & 75 & 74 & 73 & 72 & 71 & 70 & 69 & 68 & 67\end{array}$

(A) the experimental ${ }^{13} \mathrm{C}$ spectrum with all resonances of $\mathbf{1}$, and (B) an expansion of the spectral window from 66 to $105 \mathrm{ppm}$. The traces represent the ${ }^{13} \mathrm{C}$ spectrum (a), the ${ }^{13} \mathrm{C}$ APT NMR spectrum (b), and the line-fitted (“calculated") spectrum (c). 
S3. The NMR Spectrum of Kaempferol-3-O-robinoside-7-O-glucoside (1) ( ${ }^{1} \mathrm{H} 600$ $\mathrm{MHz} /{ }^{13} \mathrm{C} 150 \mathrm{MHz}$, DMSO-d 6 with $50 \%$ of $\mathrm{D}_{2} \mathrm{O}$ ): $2 \mathrm{D}{ }^{1} \mathrm{H},{ }^{1} \mathrm{H}-\mathrm{COSY}$.
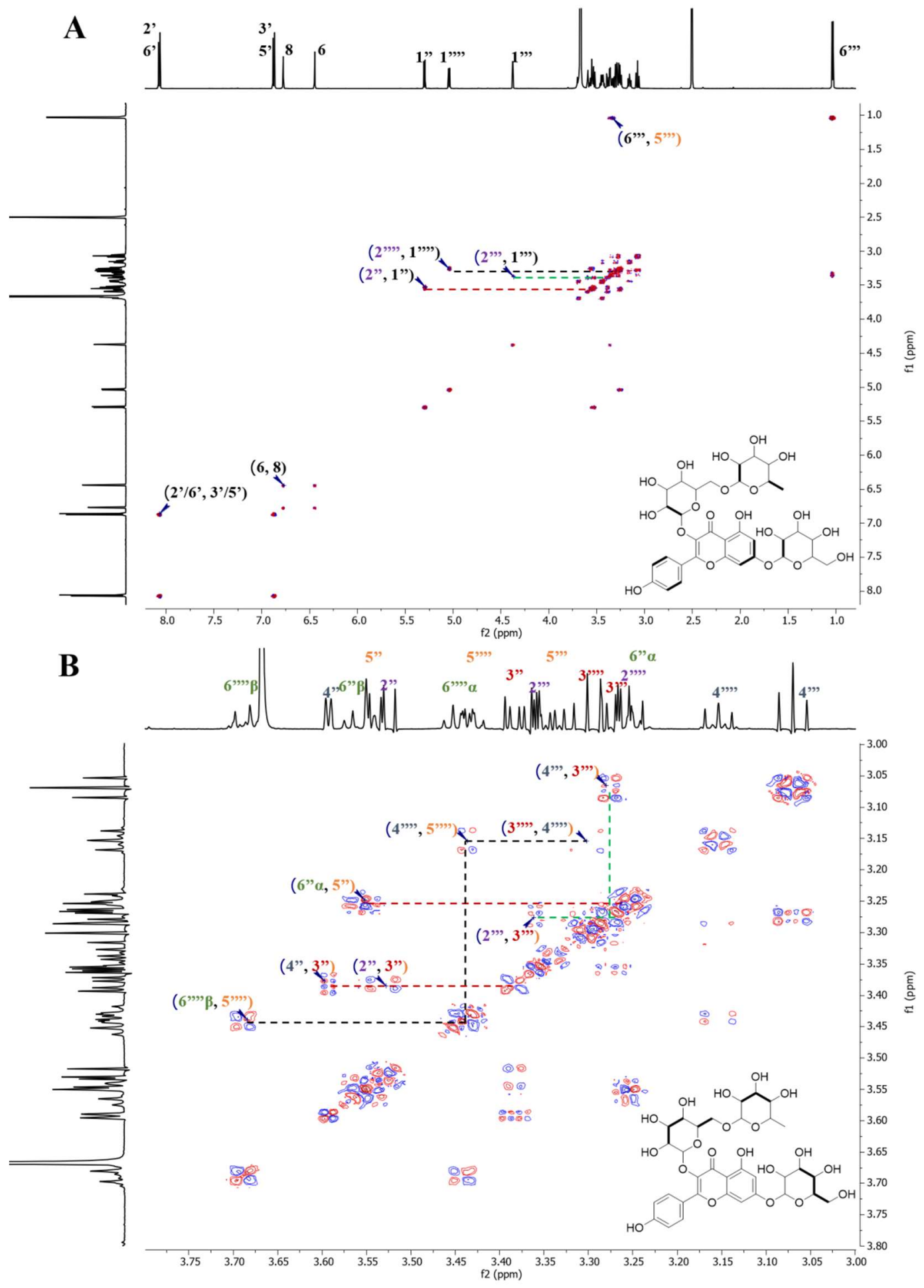

(A) the entire COSY spectrum of $\mathbf{1}$ and (B) zoomed-in spectral window. 
S4. 2D ${ }^{1} \mathrm{H},{ }^{13} \mathrm{C}$-HSQC Spectrum of Kaempferol-3-O-robinoside-7-O-glucoside (1) $\left({ }^{1} \mathrm{H}\right.$ $600 \mathrm{MHz} /{ }^{13} \mathrm{C} 150 \mathrm{MHz}$, DMSO-d 6 with $50 \%$ of $\mathrm{D}_{2} \mathrm{O}$ ).
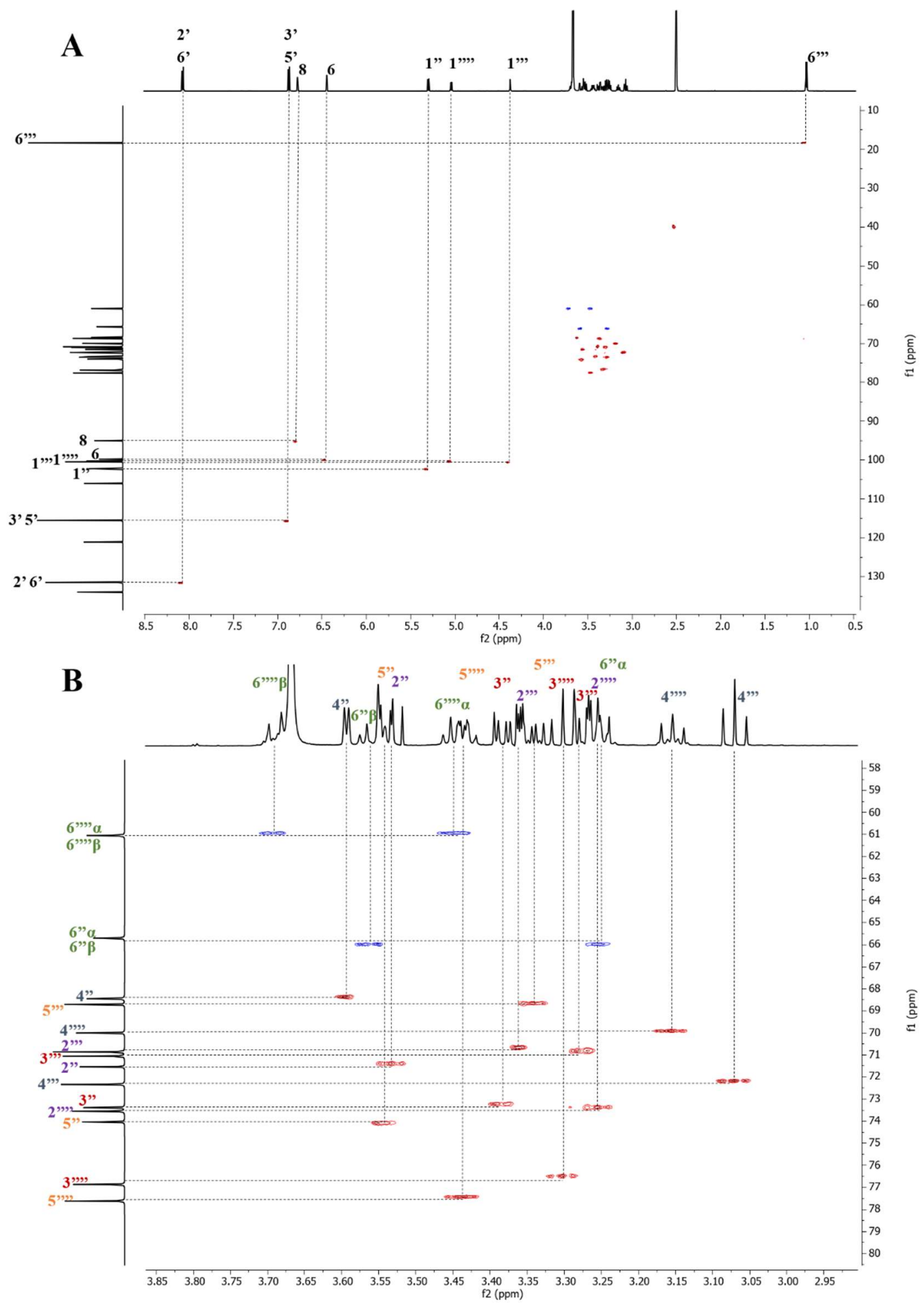

(A) the entire HSQC spectrum of $\mathbf{1}$ and (B) zoomed-in spectral window. 
S5. 2D ${ }^{1} \mathrm{H},{ }^{13} \mathrm{C}$-HMBC Spectrum of Kaempferol-3-O-robinoside-7-O-glucoside (1) $\left({ }^{1} \mathrm{H}\right.$ $600 \mathrm{MHz} /{ }^{13} \mathrm{C} 150 \mathrm{MHz}$, DMSO-d 6 with $50 \%$ of $\mathrm{D}_{2} \mathrm{O}$ ).

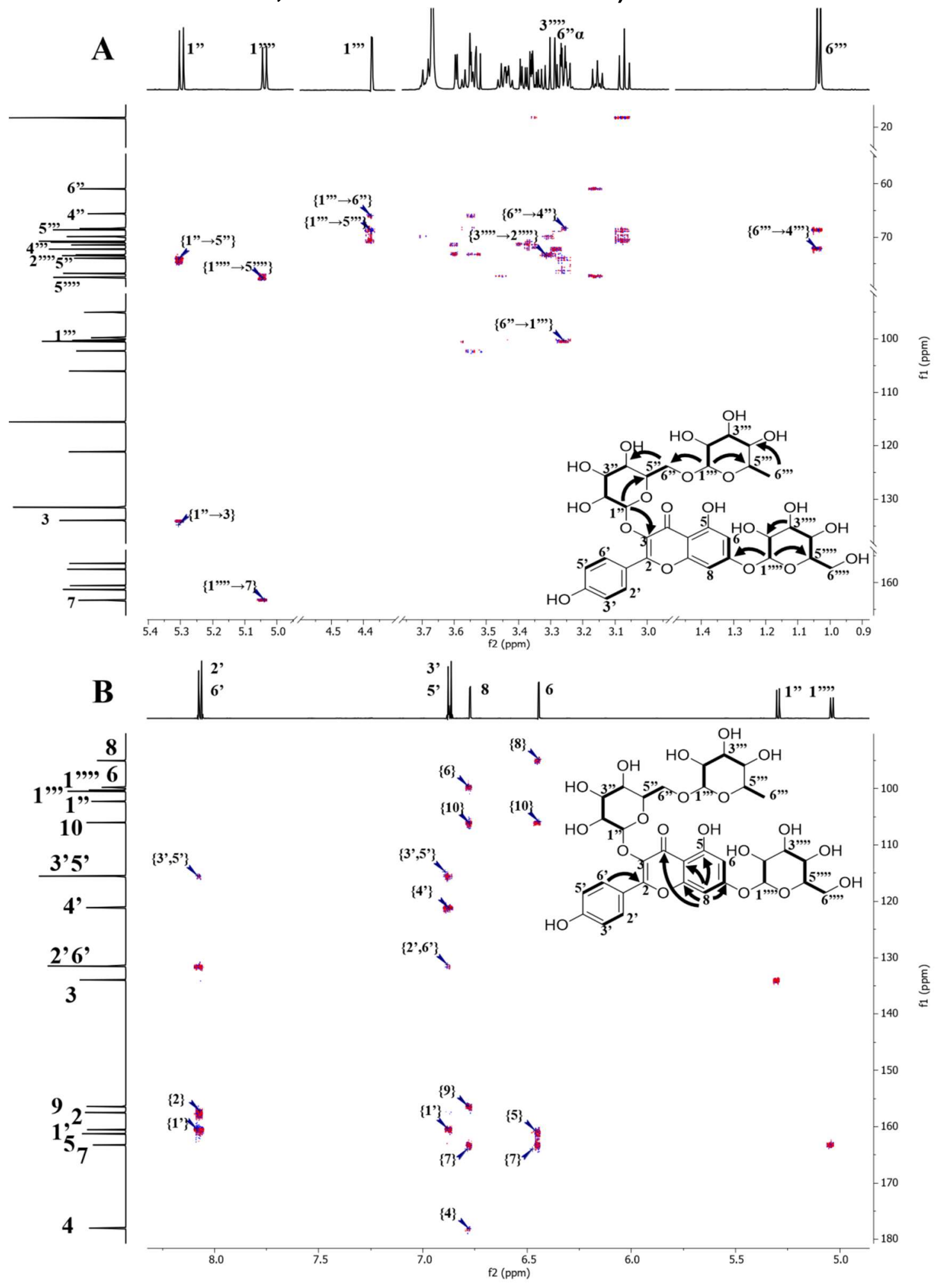

(A) the entire HMBC spectrum of $\mathbf{1}$ and (B) zoomed-in spectral window. 
S6. NMR 2D ${ }^{1} \mathrm{H},{ }^{13} \mathrm{C}-\mathrm{HMBC}$ Spectral Comparison of Kaempferol-3-O-robinoside-7-Oglucoside (1) and Quercetin-3-O-robinoside-7-O-glucoside (2) $\left({ }^{1} \mathrm{H} 400 \mathrm{MHz} /{ }^{13} \mathrm{C} 100\right.$ $\mathrm{MHz}$, DMSO-d 6 with $50 \%$ of $\mathrm{D}_{2} \mathrm{O}$ ).

A

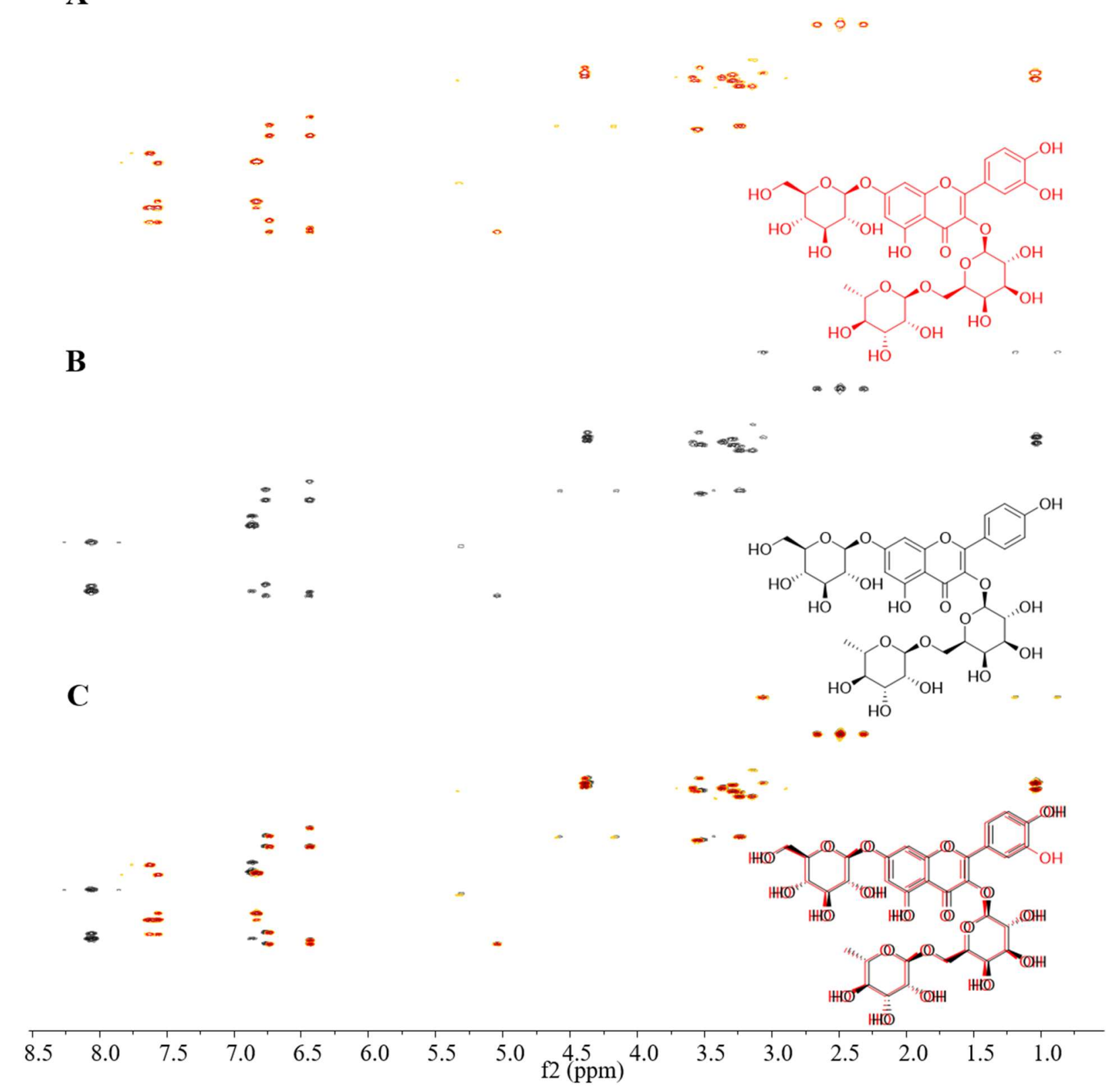

(A) the HMBC spectrum of $\mathbf{1} ;(\mathbf{B})$ the HMBC spectrum of $2 ;(\mathbf{C})$ the overlay of (A) and (B). The very close structural similarity produces two HMBC spectra that are highly congruent, yet they exhibit minor but characteristic differences that originate from the single additional $\mathrm{OH}$ group in the B-ring of quercetin in $\mathbf{2}$ vs. kaempferol in $\mathbf{1}$. All other structural characteristics, particularly for the complex and biosynthetically variable bisdesmosidic glycosidic moieties are identical in $\mathbf{1}$ and 2 and, thus, near identical in their HMBC spectra. 
S7. Numerical Comparison of the HiFSA Profiles of 1 Obtained with PERCH and CT Software Packages.

(A) Chemical shift comparison

\begin{tabular}{|c|c|c|c|}
\hline annotation & $\delta_{\mathrm{H}}(\mathrm{ppm}) / \mathrm{CT}$ & difference in chemical shiff (in $\mathrm{Hz}$, reference to $\pm 2 \mathrm{~Hz}$ scale bar) & $\delta_{\mathrm{H}}(\mathrm{ppm}) / \mathrm{PERCH}$ \\
\hline H1" & 5.2911 & \begin{tabular}{l||c|} 
& 0.02761
\end{tabular} & 5.2911 \\
\hline H1"' & 4.3691 & 0.03301 & 4.3690 \\
\hline H1"'" & 5.0357 & 0.02941 & 5.0356 \\
\hline $\mathrm{H} 2 "$ & 3.5280 & 0.03961 & 3.5280 \\
\hline H2"' & 3.3548 & 0.03181 & 3.3547 \\
\hline H2"'" & 3.2531 & 0.02701 & 3.2531 \\
\hline $\mathrm{H} 2^{\prime}-6^{\prime}$ & 8.0668 & 0.01740 & 8.0668 \\
\hline H3" & 3.3812 & 0.04081 & 3.3811 \\
\hline H3"' & 3.2699 & 0.03241 & 3.2699 \\
\hline H3"'" & 3.2989 & 0.03421 & 3.2989 \\
\hline H3'-5' & 6.8720 & 0.04441 & 6.8719 \\
\hline H4" & 3.5900 & 0.03301 & 3.5900 \\
\hline H4"' & 3.0670 & 0.03541 & 3.0669 \\
\hline H4"'" & 3.1515 & 0.02401 & 3.1515 \\
\hline H5" & 3.5408 & 0.01800 & 3.5407 \\
\hline H5"' & 3.3360 & 0.02761 & 3.3359 \\
\hline H5"'" & 3.4336 & 0.04561 & 3.4335 \\
\hline H6 & 6.4434 & 0.03841 & 6.4433 \\
\hline H6"' & 1.0291 & 0.03781 & 1.0290 \\
\hline H6"a & 3.5546 & 0.12303 & 3.5544 \\
\hline H6"'"a & 3.6867 & 0.03841 & 3.6867 \\
\hline H6"b & 3.2513 & 0.06901 & 3.2512 \\
\hline H6"'"b & 3.4441 & 0.03601 & 3.4440 \\
\hline $\mathrm{H} 8$ & 6.7731 & 0.03241 & 6.7731 \\
\hline
\end{tabular}


(B) $J$ coupling comparison

\begin{tabular}{|c|c|c|c|c|c|}
\hline \multicolumn{2}{|c|}{ Coupled Hs } & $J$ in $\mathrm{Hz} / \mathrm{CT}$ & \multicolumn{2}{|c|}{ difference in $J$ in $\mathrm{Hz}$ (reference to $\pm 2 \mathrm{~Hz}$ scale bar) } & $J$ in $\mathrm{Hz} / \mathrm{PERCH}$ \\
\hline 6 & 8 & 2.162 & & 0.011 & 2.151 \\
\hline $2^{\prime} / 6^{\prime}$ & $2^{\prime} / 6^{\prime}$ & 2.66 & & 0.047 & 2.6133 \\
\hline $2^{\prime} / 6^{\prime}$ & $3^{\prime} / 5^{\prime}$ & 8.68 & & 0.002 & 8.6783 \\
\hline $3^{\prime} / 5^{\prime}$ & $2^{\prime} / 6^{\prime}$ & 0.192 & & -0.206 & 0.3984 \\
\hline $3^{\prime} / 5^{\prime}$ & $3^{\prime} / 5^{\prime}$ & 2.43 & 1 & -0.055 & 2.485 \\
\hline $1 "$ & $2 "$ & 7.718 & & -0.010 & 7.728 \\
\hline $1 "$ & $5^{\prime \prime}$ & -0.098 & 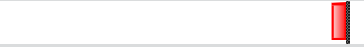 & -0.062 & -0.036 \\
\hline $2^{\prime \prime}$ & $3 "$ & 9.581 & & 0.011 & 9.570 \\
\hline $3 "$ & $4 "$ & 3.400 & & 0.003 & 3.397 \\
\hline $4 "$ & $5^{\prime \prime}$ & 0.899 & & 0.760 & 0.139 \\
\hline $4 "$ & $6 " \mathrm{lb}$ & -0.733 & & -0.387 & -0.346 \\
\hline $5^{\prime \prime}$ & $6 " \mathrm{a}$ & 5.774 & & 0.062 & 5.712 \\
\hline $5^{\prime \prime}$ & $6 " \mathrm{~b}$ & 6.730 & & -0.232 & 6.962 \\
\hline $6 " \mathrm{a}$ & $6 " \mathrm{~b}$ & -10.609 & & 0.132 & -10.741 \\
\hline $1 " '$ & $2^{\prime \prime \prime}$ & 1.631 & & 0.025 & 1.606 \\
\hline $1^{\prime \prime \prime}$ & $5^{\prime \prime \prime}$ & -0.573 & & 0.051 & -0.624 \\
\hline $2^{\prime \prime \prime}$ & $3^{\prime \prime \prime}$ & 3.427 & & 0.025 & 3.402 \\
\hline $3^{\prime \prime \prime}$ & $4 " '$ & 9.424 & & -0.004 & 9.428 \\
\hline 4"' & $5 " '$ & 9.426 & & 0.012 & 9.414 \\
\hline $4 " '$ & 6"' & -0.010 & & -0.044 & 0.034 \\
\hline $5 " '$ & 6"' & 6.202 & & 0.005 & 6.197 \\
\hline $1^{\prime \prime \prime \prime}$ & $2^{\prime \prime \prime \prime}$ & 7.796 & & -0.003 & 7.800 \\
\hline $2^{\prime \prime \prime \prime}$ & $3^{\prime \prime \prime \prime}$ & 9.114 & & -0.001 & 9.115 \\
\hline $3^{\prime \prime \prime \prime}$ & $4 " ' "$ & 8.991 & & -0.018 & 9.009 \\
\hline $4^{\prime \prime \prime \prime}$ & $5^{\prime \prime \prime}$ & 9.670 & ] & 0.046 & 9.624 \\
\hline $4^{\prime \prime \prime \prime}$ & $6 " \mathrm{l} a$ & -0.265 & & -0.030 & -0.236 \\
\hline $5^{\prime \prime \prime \prime}$ & $6 " ' \mathrm{a}$ & 6.169 & & 0.043 & 6.126 \\
\hline $5^{\prime \prime \prime}$ & $6 " ' \mathrm{~b}$ & 2.200 & & -0.001 & 2.202 \\
\hline
\end{tabular}


(C) Line width comparison

\begin{tabular}{|c|c|c|c|}
\hline annotation & line width (in Hz)/CT & difference in line width in $\mathrm{Hz}$ (reference to $\pm 2 \mathrm{~Hz}$ scale bar) & line width (in $\mathrm{Hz}) / \mathrm{PERCH}$ \\
\hline H1" & 1.009 & 0.132 & 0.877 \\
\hline H1"' & 0.981 & 0.148 & 0.833 \\
\hline H1"'" & 1.537 & -0.093 & 1.63 \\
\hline $\mathrm{H} 2 "$ & 1.315 & 0.166 & 1.149 \\
\hline H2"' & 1.192 & 0.083 & 1.109 \\
\hline $\mathrm{H} 2^{\prime \prime \prime \prime}$ & 1.534 & 0.099 & 1.435 \\
\hline $\mathrm{H} 2^{\prime}-6{ }^{\prime}$ & 1.814 & 0.092 & 1.722 \\
\hline H3" & 1.871 & -0.232 & 2.103 \\
\hline H3"' & 2.004 & 0.189 & 1.815 \\
\hline H3"'" & 1.160 & -0.006 & 1.166 \\
\hline H3'-5' & 1.239 & 0.119 & 1.120 \\
\hline H4" & 1.467 & 0.007 & 1.460 \\
\hline H4"' & 1.534 & 0.014 & 1.520 \\
\hline H4"'" & 1.687 & -0.564 & 2.251 \\
\hline H5" & 1.841 & -0.392 & 2.233 \\
\hline H5"' & 1.731 & -0.403 & 2.134 \\
\hline H5"'" & 1.534 & 0.171 & 1.363 \\
\hline H6 & 1.122 & 0.039 & 1.083 \\
\hline H6"' & 1.261 & 0.071 & 1.190 \\
\hline H6"a & 1.235 & 0.065 & 1.170 \\
\hline H6"'"a & 1.607 & 0.177 & 1.430 \\
\hline H6"b & 1.372 & -0.138 & 1.510 \\
\hline H6"'"b & 1.021 & 0.018 & 1.003 \\
\hline $\mathrm{H} 8$ & 1.159 & -0.014 & 1.173 \\
\hline
\end{tabular}

